\title{
A unique cause of small bowel obstruction
}

\author{
N. McMurray \\ F.R.C.S.
}

\author{
A. C. V. Maltby \\ F.R.C.S.
}

Tyrone County Hospital, Omagh, County Tyrone

\section{Summary}

A unique case of small bowel obstruction in an 83-year-old man is reported.

The obstruction was produced by a carcinoma of the caecum. The caecum was lying in the scrotal sac of a large indirect inguinal hernia.

\section{Introduction}

An inguinal hernia is a common surgical condition. The hernial sac may be empty or contain omentum or small bowel. Less commonly it may contain large bowel, bladder, ovary or Meckel's diverticulum. Carcinoma is a very rare finding in an organ in a hernial sac.

\section{Case report}

An 83-year-old man was admitted with a large bilateral inguino-scrotal hernia which had been present for many years.

His general health had been good and he was active for his age. His past medical history did not contain anything of relevance.

He complained of a colicky pain in the left scrotal hernia for $24 \mathrm{hr}$ before admission. He had vomited several times. On examination he was in slight discomfort and was not dehydrated or anaemic. He was apyrexial but had tachycardia of $110 / \mathrm{min}$. His abdomen was soft. The left inguinal hernia was slightly tender. There was no evidence of inflammation but the hernia was irreducible.

The right scrotal hernia was not tender and was irreducible. The abdominal bowel sounds were increased in frequency, absent over the left hernia and present over the right hernia. A plain X-ray of the abdomen revealed several dilated loops of small bowel.

A provisional diagnosis of obstructed left inguinal hernia was made-possibly of a Richter's type in view of the mildness of his symptoms.

As the left hernia was tender it was explored first via an inguino-scrotal incision. Surprisingly, the large and small bowel it contained were not in any way abnormal.
The right scrotum was therefore explored by an inguino-scrotal approach. The caecum was lying in the hernial sac and contained a carcinoma of $6 \times 6 \mathrm{~cm}$ which had penetrated the serosa. It was lightly adherent to the scrotal walls and several loops of slightly dilated small bowel were adherent to the neoplasm. There were no fistulae.

\section{Procedure}

The small bowel was released, the caecum was dissected from the scrotum and excised. Bowel continuity was established by end-to-end anastomosis of ileum to ascending colon. The hernial sac was excised and the area repaired. Metastases were noted in the small bowel mesentery and in the liver.

Apart from a chest infection the postoperative course was satisfactory but on the 16th postoperative day he became drowsy and confused and died on the next day. The cause of death was pulmonary embolism.

\section{Discussion}

A review of the English language bibliography of the past 25 years has not revealed any reports of small bowel obstruction related to a caecal carcinoma lying in the sac of an inguinal hernia.

Fieber and Wolstenholm (1955) have suggested that irreducibility of an inguinal hernia in an elderly patient should raise the suspicion of a co-existing neoplasm in the hernial sac.

In the present case, although the hernia was present for $>\mathbf{2 0}$ years and irreducible, there was no clinical evidence of caecal neoplasia, namely weight loss, lassitude, anaemia and dyspnoea.

Silberman (1969) reported a caecal carcinoma in an inguinal hernia which presented as a scrotal abscess, the precipitating factor being blunt trauma to the scrotum. His patient was grossly anaemic $(7 \cdot 4 \mathrm{~g} / \mathrm{dl})$ but had no signs of small gut obstruction.

The present patient was perhaps fortunate to have developed his obstruction as otherwise he might not have sought medical attention until the caecal neoplasm had enlarged sufficiently to obstruct 
the bowel or he had developed the symptoms of anaemia.

The authors cannot explain the presence of tenderness in the left scrotum and the lack of signs over the right scrotum.

Carcinoma of caecum may not be as silent as it has been frequently described in the textbooks.

Forte and Cohn (1970) analysed 139 cases of caecal neoplasm over the period 1948-1963. They found the typical presentation was abdominal pain or discomfort, weight loss and a palpable mass in $87 \%$. Twenty per cent. had symptoms of partial small bowel obstruction and only $17 \%$ were correctly diagnosed before laparotomy. They noted that symptoms were frequently known to patients for 3 several months or even one year before medical $\stackrel{\Phi}{\complement}$ attention was sought.

They noted that $22.5 \%$ of patients with colonic carcinoma had an inguinal hernia or had hernial repairs carried out in the preceding 2 years. This association is statistically significant.

\section{References}

Fieber, S.S. \& Wolstenholme, J.T. (1955) Primary tumours ڤ in inguinal hernial sacs. Archives of Surgery, 71, 254.

FORTE, C.E. \& COHN, I. (1970) Cancer of cecum-review of 139 cases. American Surgeon, 36, 129.

Silberman, V.A. (1969) Caecal carcinoma incarcerated in an inguinal hernia. International Surgery, 51, 515. 